\title{
Transverse kink oscillations of expanding coronal loops
}

\author{
I. Ballai and B. Orza
}

\begin{abstract}
Solar Physics and Space Plasma Research Centre $\left(\mathrm{SP}^{2} \mathrm{RC}\right)$, Department of Applied Mathematics, The University of Sheffield, Sheffield, S3 7RH, UK

e-mail: [i.ballai;b.orzai]@sheffield.ac.uk
\end{abstract}

Received 22 May 2012 / Accepted 24 July 2012

\begin{abstract}
Aims. We investigate the nature of transverse kink oscillations of loops expanding through the solar corona and examine how can oscillations be used to diagnose plasma parameters and the magnetic field. In particular, we aim to analyse how the temporal dependence of the loop length (here modelling the expansion) will affect the $P_{1} / P_{2}$ period ratio of transverse loop oscillations. Methods. Due to the uncertainty of the loop's shape through its expansion, we discuss separately the case of the loop that maintains its initial semi-circular shape and the case of the loop that evolves into an elliptical-shape loop from a semi-circular shape. The equations that describe the oscillations in an expanding flux tube are complicated due to the spatial and temporal dependence of coefficients. Using the WKB approximation, we find approximative values for periods and their evolution as well as the period ratio. For small values of time (near the start of the expansion), we can employ a regular perturbation method to find approximative relations for eigenfunctions and eigenfrequencies.

Results. Using simple analytical and numerical methods, we show that the period of oscillations are affected by the rising of the coronal loop. The change in the period due to the increase in the loop's length is more pronounced for loops that expand into a more structured (or cooler) corona. The deviation of periods will have significant implications in determining the degree of stratification in the solar corona. The effect of expansion on the periods of oscillations is considerable only when the loop is expanding but not after it has reached its final stage.

Conclusions. The present study improves our understanding of the complexity of dynamical processes in the solar corona, in particular the changes of periods of kink oscillations due to temporal changes in the characteristics of the coronal loop. Our results clearly show that the problem of expansion of coronal loops can introduce significant changes in the period of oscillations, with consequences on the seismological diagnostics of the plasma and magnetic field.
\end{abstract}

Key words. magnetohydrodynamics (MHD) - Sun: corona - Sun: oscillations

\section{Introduction}

Dynamical processes and transients observed in solar and space plasmas have received considerable attention due to their ability to help scientists to diagnose remotely the parameters of the medium (temperature, scale-height, density, transport coefficients, etc.) in which these events occur, the magnitude and structure of the magnetic field, and the stability of the plasma. Seismological techniques and methods, imported from Earth's seismology and helioseismology, assume the combination of high-resolution observations (amplitude, wavelength, propagation speed, damping time/length) with theoretical models (dispersion and evolutionary equation) to derive quantities that cannot be measured directly or indirectly. In particular, coronal seismology has emerged as one of the most dynamically developing methods of solar physics (see, e.g. Roberts et al. 1984; Nakariakov et al. 1999; Ruderman \& Roberts 2002; Andries et al. 2005, 2009; Ballai et al. 2005; Banerjee et al. 2007; Verth et al. 2007; Ballai 2007; Ruderman et al. 2008; Morton \& Erdélyi 2009; Ruderman \& Erdélyi 2009; Wang et al. 2012, etc.).

One important candidate in coronal seismology are transverse kink oscillations, i.e. oscillations that exhibit periodic movement about the loop's symmetry axis. Recent Coronal Multi-Channel Polarimeter (CoMP) observations (Tomczyk et al. 2007) showed that the predominant motion of coronal loops is the transverse kink oscillation, which is also the easiest to generate. The triggering of these oscillations can be through a lateral forcing process at arbitrary height of the loop by a blast wave (or EIT wave) that emanates as a result of a sudden energy release by a flare and/or CME (see, e.g. Ballai 2007; Ballai et al. 2008). Kink oscillations can also be triggered by the transverse motion of footpoints due to the granular buffeting of flux tubes in the photosphere. This scenario is true not only for coronal structures, but also for all magnetic entities in the solar atmosphere that can serve as waveguides. Global EIT waves can additionally interact with prominence fibrils to generate kink waves and oscillations in prominences, as observed by, e.g. Ramsey \& Smith (1966) and more recently by Eto et al. (2002), Jing et al. (2003), Okamoto et al. (2004), Isobe \& Tripathi (2007), and Pintér et al. (2008) (for a comprehensive review see, e.g. Arregui et al. 2012).

The dispersion relations for many simple (and some quite complicated) plasma waves under the assumptions of ideal magnetohydrodynamics (MHD) are well known; they were derived long before accurate EUV observations were available (see, e.g. Edwin \& Roberts 1983; Roberts et al. 1984) using simplified models within the framework of ideal and linear MHD. Although the realistic interpretation of many observations is made difficult by the insufficient spatial and temporal resolution of present-day satellites, considerable information about the state of the plasma and the structure and magnitude of the coronal magnetic field can still be obtained.

The mathematical description of waves and oscillations in solar structures is, in general, given by equations whose coefficients vary in space and time. It has been recognised by, e.g. Andries et al. (2005) that the longitudinal stratification (i.e. 
along the longitudinal symmetry axis of the tube that coincides with the direction of the magnetic field) is modifying the periods of oscillations of coronal loops. Accordingly, in the case of kink waves, these authors showed that the ratio $P_{1} / P_{2}$ (where $P_{1}$ refers to the period of the fundamental transverse oscillation, while $P_{2}$ describes the period of the first overtone of the same oscillation) can differ, sometimes considerably, from the canonical value of 2 , that would be recovered if the loops were homogeneous. These authors also showed that the deviation of $P_{1} / P_{2}$ from 2 is proportional to the degree of stratification. This problem was also discussed in other studies, such as Dymova \& Ruderman (2006), Diaz et al. (2007), McEwan et al. (2008), Ballai et al. (2011), Orza et al. (2012), etc. In a recent analysis, Ballai et al. (2011) discusses the ambiguity of the period ratio seismology, since other effects could result in the observation of multiple periods and each interpretation yields in different value for the magnetic field and/or degree of stratification.

Studies by, e.g. Verth et al. (2007) later showed that not only density stratification can modify the $P_{1} / P_{2}$ period ratio as the variation of the loop's cross section area also has an effect on the period ratio. While density stratification tends to decrease the period ratio, a modification of the cross section (i.e. when the magnetic field is flaring up as we approach the apex) tends to increase the $P_{1} / P_{2}$ value.

The investigation of properties of oscillations of coronal loops when equilibrium parameters of the plasma depend on time is a relatively new area of dynamical studies in the solar atmosphere, in particular for coronal seismology. Morton \& Erdélyi (2009) and Morton et al. (2010) studied the effect of cooling (i.e. temporal dependence of temperature) on the dynamics of kink oscillations and travelling waves. They found that the cooling of the plasma results in period decrease and amplification of oscillations and is thus a mean to dissipate the energy stored in waves propagating in an unbounded plasma. The same idea was used later by Morton et al. (2011) to study the properties of torsional Alfvén waves in coronal loops. The problem was recently reconsidered by Ruderman (2011a), who showed that the cooling also generates an amplification of kink oscillations. This amplification appears to be a competing effect with the damping due resonant absorption, as shown by Ruderman (2011b).

The problem of loop emergence and expansion through the solar atmosphere is one of the most challenging topics of solar physics because it involves analysing of the evolution of the magnetic field in different regions of the solar interior and atmosphere, where conditions can change from region to region. According to the standard theory, the magnetic field produced by the dynamo action in the tachocline is transported through the solar convective zone towards the solar surface by magnetic buoyancy coupled with convective motion (Parker 1955, 1988). Once at the surface, the emerged flux tube creates sunspots and bipolar active regions (Zwaan 1987). In the solar atmosphere, the rise of the flux tube continues due to an excess of the magnetic pressure inside the loop (e.g. Archontis et al. 2004). For the purpose of our investigation, we will assume that this excess is balanced at the transition region (TR) and that from this height the expansion is no longer a driven problem instead, the loop moves through the corona in the virtue of its inertia. During the emergence and expansion phase, the flux tube can interact with existing magnetic structure in the solar atmosphere, which might be responsible for the appearance of small-scale (e.g. compact flares, plasmoids, X-point brightenings) and large-scale events (flares and CMEs), as suggested by Archontis (2004). Loop emergence often is associated with strong upflows, as observed by, e.g. Harra et al. (2010, 2012).

It is straightforward to imagine what happens with oscillations in a loop when the length of the loop is increasing. As the length becomes larger, the frequency of oscillations becomes smaller, i.e. the periods of oscillations are expected to grow. In an inhomogeneous waveguide, however, particular periods will be differently affected by the combined effect of inhomogeneity and loop length increase. Therefore, we expect that the period ratio of oscillations will change not only with the degree of inhomogeneity but also with time.

The aim of this paper is to investigate the effect of loop expansion through the solar corona on the period ratio $P_{1} / P_{2}$ and the consequences of the inclusion of the length of the loop as a dynamical parameter on estimations of the degree of density stratification. The paper is structured in the following way: in Sect. 2, we introduce the mathematical formalism and obtain analytical results for an expanding loop that initially starts from a semi-circular shape, which is preserved throughout the expansion. In Sect. 3, we generalise our findings by assuming that the expansion of the loop in the solar corona does not occur with the same speed in the horizontal and vertical direction. In this case, the initial semi-circular loop transforms into a loop with elliptic shape. Our results are summarised in the last section.

\section{The mathematical formulation of the problem}

In our analysis, we will capture the dynamical behaviour of the loop once it has reached the transition region (TR). At this height, the loop can interact with a blast wave propagating in the low solar corona. This instigator could be easily identified with an EIT wave that propagates in the low corona over very large distances. EIT waves are known to be one of the major sources for kink oscillations of coronal loops (see, e.g. Ballai 2007). We assume that the height at which the loop starts its journey through the corona is at $3 \mathrm{Mm}$ above the solar surface. A typical loop length is of the order of $300 \mathrm{Mm}$. For practical reasons, we consider that the height of the loop in its final position would be about 97, Mm resulting in a loop length of about $305 \mathrm{Mm}$.

The raising speed of loops is generally taken to be 10-15 $\mathrm{km} \mathrm{s}^{-1}$ (Chou \& Zirin 1988; Archontis 2008). Consequently, the time needed for the loop to travel the distance from the TR to its steady position can easily be estimated to be between 3.4 and $5.2 \mathrm{~h}$. This time is at least two orders of magnitude larger than a typical period of kink oscillations, so there is enough time for the development of oscillations. Higher rising speeds are also possible. Recently, Schmidt \& Ofman (2011) reported expansions of a post-flare loop with speeds of hundreds $\mathrm{km} \mathrm{s}^{-1}$. Standing waves are formed if the speed of change in the length of the loop is smaller than the period of oscillations. This condition is easily satisfied for fast kink oscillations. We assume that the loop expands into the "empty" corona, i.e. it will not encounter any interaction with existing magnetic elements.

In the first instance, we assume that the semi-circular shape of the loop at the TR is preserved throughout the expansion. Assuming an initial height of $3 \mathrm{Mm}$ above the surface, the distance between the footpoints of the loop is $6 \mathrm{Mm}$. When reaching the final height of $190 \mathrm{Mm}$, the footpoints travel over a distance of $197 \mathrm{Mm}$. In addition, we assume that the expansion occurs at constant temperature (isothermal process) and that the expansion the loop maintains its cross section constant throughout the expansion. Due to the increase in the volume of the loop, a pressure difference is generated. This means that the plasma flows along magnetic field lines, resulting in a density that depends 
not only on the height, $z$, but also on time. As it raises through the solar corona, the tangent to the loop is also changing monotonically. Therefore, the momentum equation in an equilibrium state becomes

$\rho_{0} \frac{\partial u}{\partial t}+\rho_{0} u \frac{\partial u}{\partial z}=-\frac{\partial p_{0}}{\partial z}-g \rho_{0} \cos \beta$

where the angle $\beta$ is a function depending on time and space, $u$ is the equilibrium flow of the plasma, $\rho_{0}$ and $p_{0}$ are the equilibrium density and pressure, and $g$ is the gravitational acceleration. In coronal loops, the flows are of the order of a few tens of $\mathrm{km} \mathrm{s}^{-1}$. As a result, in Eq. (1) we can neglect the terms on the left-hand side because

$u \frac{\partial u}{\partial z} \sim \frac{u^{2}}{L} \approx \frac{10^{8}}{10^{8}}=O(1)$,

that is, two order of magnitude smaller than the terms on the right-hand side. We can write pressure as

$p_{0}(z, t)=\frac{k_{\mathrm{B}} T_{0} \rho_{0}(z, t)}{m}$,

where $k_{\mathrm{B}}$ is the Boltzman constant, $T_{0}$ is the constant temperature and $m$ is the mean atomic mass per particle. Introducing this expression into the RHS of Eq. (1), we obtain that

$\frac{1}{\rho_{0}} \frac{\partial \rho_{0}}{\partial z}+\frac{\cos \beta(z, t)}{H}=0$,

where $H$ is the constant density scale-height. Focussing on the density distribution inside the loop, we can integrate the above equation to obtain

$\rho_{\mathrm{i}}=\rho_{\mathrm{f}} \exp \left[-\int_{0}^{z} \frac{\cos \beta\left(z^{\prime}, t\right)}{H} \mathrm{~d} z^{\prime}\right]$.

Assuming that the loop is semi-circular, we obtain that $\beta(z, t)=$ $\pi z / L(t)$, so that the density inside the coronal loop becomes

$\rho_{\mathrm{i}}=\rho_{\mathrm{f}} \exp \left[-\frac{L(t)}{\pi H} \sin \frac{\pi z}{L(t)}\right]$,

where $\rho_{\mathrm{f}}$ is the density of the plasma at the footpoint of the loop. For simplicity we will assume that the external density can simply be written as $\rho_{\mathrm{e}}=D \rho_{\mathrm{i}}$, where throughout our calculations, we consider $D=0.5$. Figure 1 depicts schematically the change of the equilibrium density, both in space and time. Here, length was normalised to the length of the loop at the start of the emergence (considered $L_{0}=3 \pi \mathrm{Mm}$ ), and time (here denoted by $\tau$ ) was normalised to the quantity $L_{0} / v_{r}$, where $v_{r}$ is the vertical rising speed of the loop considered $v_{r}=15 \mathrm{~km} \mathrm{~s}^{-1}$. According to our expectations, the density of the loop decreases with time. We assume that the flux tube is thin, even at the beginning of its expansion.

In the thin flux tube approximation, the dynamics of transverse kink oscillations is given by (see, e.g. Ruderman 2010 , 2011a)

$\rho_{\mathrm{i}}\left(\frac{\partial}{\partial t}+U_{\mathrm{i}} \frac{\partial}{\partial z}\right)^{2} \eta+\rho_{\mathrm{e}}\left(\frac{\partial}{\partial t}+U_{\mathrm{e}} \frac{\partial}{\partial z}\right)^{2} \eta-\frac{2 B^{2}}{\mu_{0}} \frac{\partial^{2} \eta}{\partial z^{2}}=0$,

where $U_{\mathrm{i}}$ and $U_{\mathrm{e}}$ are the internal and external flows, $B$ is the magnetic field strength (here assumed to have identical value inside and outside the loop), $\mu_{0}$ is the magnetic permeability of the free space, and $\eta$ is a complex valued displacement of the
Density profile

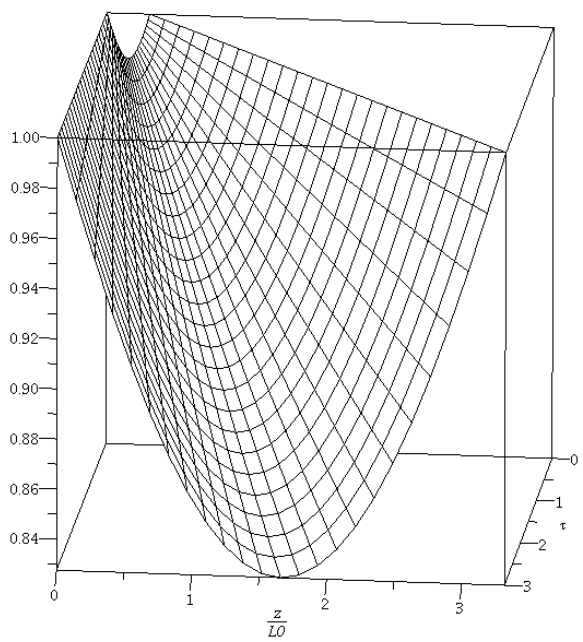

Fig. 1. Schematic representation of the evolution of the equilibrium density measured on the vertical axis in the units of density at the footpoint. Here, lengths are given in units of the loop length at the start of the expansion in the corona $\left(L_{0}\right)$, and time is given in units of $L_{0} / v_{r}$, where $v_{r}$ is the constant rising speed in the vertical direction (here taken to be $15 \mathrm{~km} \mathrm{~s}^{-1}$ ).

loop, with $\eta=\eta_{R}+\mathrm{i} \eta_{I}$. In Cartesian coordinates, the loop's displacement in the $x$ and $y$ directions, $\xi_{x}$ and $\xi_{y}$, is given by

$\xi_{x}=\eta_{R}, \quad \xi_{y}=-\eta_{I}$.

We assume a quasi-stationary equilibrium, so that the characteristic time variation of equilibrium quantities $\left(t_{\mathrm{ch}}\right)$ is much longer than the period of kink oscillations $(P)$, and introduce the small parameter $\epsilon$, so that $P=\epsilon t_{\mathrm{ch}}$. Using the definition of the period of oscillations, we can write

$\frac{L\left(\mu_{0} \rho_{\mathrm{ch}}\right)^{1 / 2}}{B_{0}}=\epsilon t_{\mathrm{ch}} \Longrightarrow B=\epsilon^{-1} \frac{L\left(\mu_{0} \rho_{\mathrm{ch}}\right)^{1 / 2}}{t_{\mathrm{ch}}}$,

meaning that we can introduce a scaled magnetic field, so that $\tilde{B}_{0}=\epsilon B_{0}$. As a result, the equation describing the dynamics of the kink oscillations can be written as

$\rho_{\mathrm{i}}\left(\frac{\partial}{\partial t}+U_{\mathrm{i}} \frac{\partial}{\partial z}\right)^{2} \eta+\rho_{\mathrm{e}}\left(\frac{\partial}{\partial t}+U_{\mathrm{e}} \frac{\partial}{\partial z}\right)^{2} \eta-\frac{2 \epsilon^{-2} \tilde{B}^{2}}{\mu_{0}} \frac{\partial^{2} \eta}{\partial z^{2}}=0$.

The above equation must be solved subject to standard boundary conditions, i.e.

$\eta(z=0, z=L)=0$.

Following the solution method proposed by Ruderman (2011a), we will solve Eq. (7) using the Wentzel-Kramers-Brillouin (WKB) method (see, e.g. Bender \& Ország 1987) and assume that the solution of the equation will be of the form

$\eta=\sum_{k=0}^{\infty} \epsilon^{k} S_{k}(z, t) \exp \left[\frac{\mathrm{i}}{\epsilon} \Phi(t)\right]$.

In the first order of approximation (often called geometric optics), Eq. (7) reduces to

$\frac{\partial^{2} S_{0}}{\partial z^{2}}+\frac{\Omega^{2}}{\tilde{c}_{\mathrm{K}}^{2}} S_{0}=0$ 
where

$\Omega=\frac{\mathrm{d} \Phi(t)}{\mathrm{d} t}, \quad \tilde{c}_{\mathrm{K}}^{2}=\frac{2 \tilde{B}^{2}}{\mu_{0}\left(\rho_{\mathrm{i}}(z, t)+\rho_{\mathrm{e}}(z, t)\right)}$.

Equation (9) must be solved subject to the boundary condition $S_{0}=0$ when $z=0$ and $z=L$. Together with the line-tying condition, Eq. (9) forms an eigenvalue problem, in which $\Omega$ is the eigenvalue and $\Omega^{2}$ is a real function.

In the next order of approximation (also called the approximation of physical optics), Eq. (7) reduces to

$\frac{\partial^{2} S_{1}}{\partial z^{2}}+\frac{\Omega^{2}}{\tilde{c}_{\mathrm{K}}^{2}} S_{1}=\frac{2 \mathrm{i} \Omega}{\tilde{c}_{\mathrm{K}}^{2}}\left[\frac{\partial S_{0}}{\partial t}+\frac{S_{0}}{2 \Omega} \frac{\mathrm{d} \Omega}{\mathrm{d} t}+\frac{\rho_{\mathrm{i}} U_{\mathrm{i}}+\rho_{\mathrm{e}} U_{\mathrm{e}}}{\rho_{\mathrm{i}}+\rho_{\mathrm{e}}} \frac{\partial S_{0}}{\partial z}\right]$,

which has to be solved subject to the boundary condition $S_{1}(z=$ $0, z=L)=0$. The boundary-value problem determining $S_{1}$ has a solution only when the RHS of Eq. (10) satisfies the compatibility condition, i.e. the orthogonality to $S_{0}$. After the RHS of Eq. (10) is multiplied by $S_{0}$ and integrated with respect to $z$ in the interval $(0, L)$, the compatibility condition reduces to (similar to Ruderman 2011a)

$\omega \int_{0}^{L} \frac{S_{0}^{2}}{c_{\mathrm{K}}^{2}} \mathrm{~d} z=$ const.

where

$\omega=\epsilon^{-1} \Omega, \quad c_{\mathrm{K}}=\epsilon^{-1} \tilde{c}_{\mathrm{K}}$.

As a consequence, the dynamics of kink oscillations in coronal loops is fully described by the system of Eqs. (9) and (11). In deriving Eq. (11), we took into account the mass conservation equation, relating the plasma flow and its density

$\frac{\partial \rho}{\partial t}+\frac{\partial(\rho U)}{\partial z}=0$

Let us now discuss a special case when the density depends on time only. This case would correspond to an initial expansion of the loop, when the height of the loop is less than the scaleheight (assuming expansion into an isothermal 1 MK corona, this height would correspond to $47 \mathrm{Mm}$ ). In this case, we may expect that the amplitude of oscillations increases as the loop expands. Indeed, it is easy to show that the amplitude of oscillations behaves as

$A(t)=A(0)\left(\frac{c_{\mathrm{K}}(t)}{c_{\mathrm{K}}(0)}\right)^{1 / 2}=A(0)\left(\frac{L(t)}{L(0)}\right)^{1 / 2}$,

where $A(0), c_{\mathrm{K}}(0)$ and $L_{0}$ are the amplitude of oscillations, the kink speed and the length of the loop at $t=0$.

A particular case worth discussing separately is when the expansion of the loop occurs linearly with time and we write that

$L(t)=L_{0}+v_{r} t$,

where $L_{0}$ is the length of the loop at the initial time, i.e. at the TR level $\left(L_{0}=3 \pi \mathrm{Mm}\right)$, and $v_{r}$ is the rising speed, here assumed constant. Accordingly, the kink speed becomes

$c_{\mathrm{K}}^{2}=c_{\mathrm{Kf}}^{2} \exp \left[\frac{L_{0}+v_{r} t}{\pi H} \sin \frac{\pi z}{L_{0}+v_{r} t}\right]$,

where

$c_{\mathrm{Kf}}^{2}=\frac{2 B^{2}}{\mu_{0} \rho_{\mathrm{f}}(1+D)}$.

A118, page 4 of 8

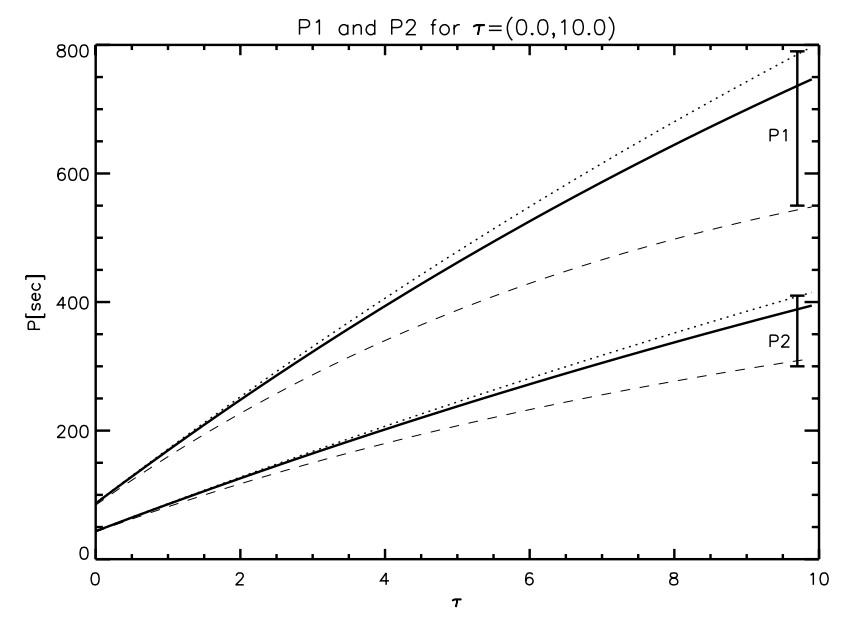

Fig. 2. Variation of the periods of the fundamental mode and its first harmonic with the dimensionless time variable $\tau$ for three different values of stratification: $H=70.5 \mathrm{Mm}$ (dotted line), $H=47 \mathrm{Mm}$ (solid line), and $H=23.5 \mathrm{Mm}$ (dashed line).

Returning to the general case, the equations describing the dynamics of kink oscillations are

$\frac{\partial^{2} S_{0}}{\partial z^{2}}+\frac{\omega^{2}}{c_{\mathrm{K}}^{2}} S_{0}=0, \quad \omega \int_{0}^{L} \frac{S_{0}^{2}}{c_{\mathrm{K}}^{2}} \mathrm{~d} z=$ const.

Let us introduce a new set of dimensionless quantities

$\xi=\frac{z}{L_{0}}, \quad \tau=\frac{v_{r} t}{L_{0}}, \quad \tilde{\omega}=\frac{\omega L_{0}}{c_{\mathrm{Kf}}}, \quad \tilde{S}_{0}=\frac{S_{0}}{L_{0}}$.

In the new variables, the equations to be solved transform into

$\frac{\partial^{2} \tilde{S}_{0}}{\partial \xi^{2}}+\exp \left[\frac{-L_{0}(1+\tau)}{\pi H} \sin \frac{\pi \xi}{1+\tau}\right] \tilde{\omega}^{2} \tilde{S}_{0}=0$,

and

$\tilde{\omega} \int_{0}^{1+\tau} \tilde{S}_{0}^{2} \exp \left[\frac{-L_{0}(1+\tau)}{\pi H} \sin \frac{\pi \xi}{1+\tau}\right] \mathrm{d} \xi=$ const.

which should be solved subject to the boundary conditions $S_{0}(\xi=0 ; \xi=1+\tau)=0$. The solution of the system (17)-(18) can be found numerically using, e.g. the shooting method. In Fig. 2, we display first the variation of periods of oscillations of the fundamental mode and its first harmonic for three different values of $H$ in terms of the dimensionless time variable, $\tau$. The bands for each period are clearly labelled in the figure. The three distinct value of periods were obtained for three values of scale-height, keeping the initial length of the loop at $3 \pi \mathrm{Mm}$. The dotted line corresponds to a scale-height of $70.5 \mathrm{Mm}$, which, assuming a plasma in hydrostatic equilibrium, would correspond to a plasma temperature of $1.5 \mathrm{MK}$. The solid line is plotted for a loop expanding into a corona where the constant scale-height is $47 \mathrm{Mm}$, corresponding to a $1 \mathrm{MK}$ hot plasma. Finally, the dashed line stands for an expansion of the loop into a plasma, where the density scale-height is $23.5 \mathrm{Mm}$, corresponding to a plasma temperature of $0.5 \mathrm{MK}$. The two bands for the periods clearly show that the two oscillations are differently affected by the expansion, i.e. change in the length of the loop. This is also obvious in Fig. 3, where we plotted the ratio of the periods of the fundamental and first harmonic as a function of the time variable $\tau$. The plot clearly shows that the oscillations of a loop expanding into a "hot" plasma (i.e. large scaleheight) are the least affected, but in all three cases, the period 


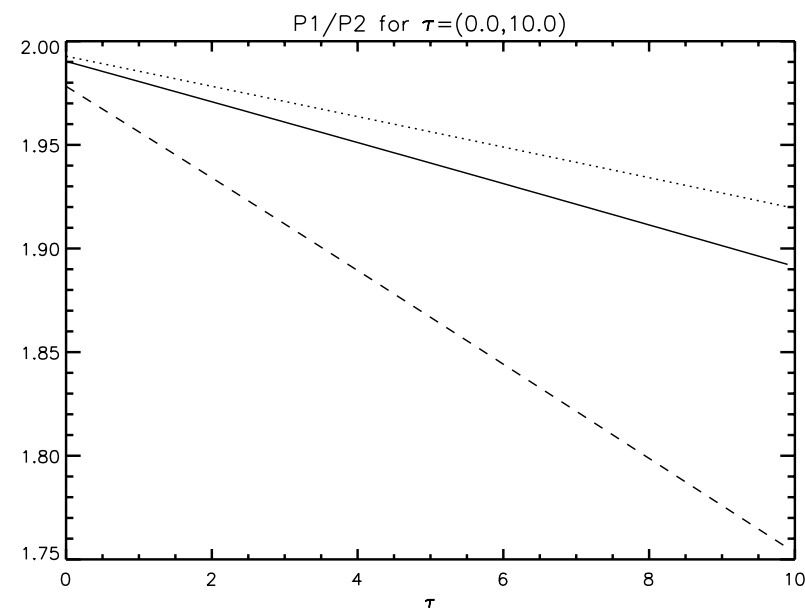

Fig. 3. Variation of the $P_{1} / P_{2}$ period ratio with respect to the dimensionless parameter $\tau$, for a loop expanding in the solar corona with persisting semi-circular shape. The meaning of each line-style is identical to Fig. 2.

ratio decreases with time. We should note here that the periods shown in Fig 2 do not start at the value of 2 because at the start of their expansion through the solar corona, they are already stratified and the least stratified is the case that corresponds to $H=1.5 \mathrm{MK}$. We ought to note that in observations, the identification of periods in coronal loops is a dynamical process, i.e. in EUV the intensity is measured in one location (or mega-pixel to reduce errors) for a long time-period. The duration of observation varies, and this duration is mostly driven either by the availability of the instrument or by the limited lifetime of oscillations before they are damped by, e.g. resonant absorption. Observations show, however, that not all loop oscillations damp (probably these oscillations are maintained by a constant lateral buffeting). Typically, for damped oscillations, the detection time is a few periods with a range of 6.7-90 min (Aschwanden 2004). In terms of the dimensionless quantity $\tau$, this range would correspond to $\tau=0.64-8.6$. Although the lower limit is too small to count in the effect of expansion, a duration of $\tau=8.6$ would add an important effect in studying the transverse kink oscillations. Figure 3 shows that for one given value of internal structuring, the value of the period ratio can also change because of the expansion of the loop. Although the periods shown in Fig. 2 display a monotonic increase with the parameter $\tau$, in reality these values will saturate, with saturation occurring faster for those modes that propagate in a highly structured plasma (e.g. for the case of $H=23.5 \mathrm{Mm}$, the saturation value of periods is about $400 \mathrm{~s}$ and saturation starts at about $\tau=20$ ). In addition, the period ratio for all cases discussed here tends to same value (near 1) for large values of $\tau$. Significantly, the effect of expansion on the periods of oscillations and the period ratio is relevant only in the expansion phase of the loop.

Analytical solutions of Eq. (17) can be obtained for the limiting case of a loop at the beginning of its expansion through the solar corona, i.e. small values of $\tau$ (see Appendix). The results confirm the tendency of periods to increase with time and of the period ratio to decrease with time.

\section{Noncircular emergence}

In reality, the expansion of a loop in the empty corona so that the semi-circular shape is preserved is unlikely since the foot points have to move in a much denser plasma than the apex of the loop. Consequently, in this section we will assume that the expansion rate in the vertical direction is larger than the expansion of footpoints in the horizontal direction. The expansion still starts at the TR level, where the shape of the loop is semi-circular and we assume that the process remains isothermal. As a result of different expansion rates, the loop evolves so that the shape becomes more elliptical. The properties of transverse loop oscillations in an elliptical coronal loop was studied recently by Morton \& Erdélyi (2009) who assumed that the semi-elliptical shape is reached in the emerging stage, before reaching a semicircular shape. Although their topic is related to the research of the present study, the problem of expansion is a dynamical process that should be treated accordingly. They found that the difference in $P_{1} / P_{2}$ period ratio between the circular and elliptical shape is up to $6 \%$.

Since the dynamics is going to be different over the two directions, it is more convenient to introduce a polar coordinate system in which

$x=a(t) \cos \theta, \quad z=b(t) \sin \theta$,

with $\dot{a}(t)<\dot{b}(t)$, the overdot denotes the derivative with respect to time, and the length of the loop is covered by the parameter $\theta$ that varies now between 0 and $\pi$. It is more convenient to use the coordinates along the loop $s$, meaning that the dynamics of transverse kink oscillations can be described by

$\rho_{\mathrm{i}}\left(\frac{\partial}{\partial t}+U_{\mathrm{i}} \frac{\partial}{\partial s}\right)^{2} \eta+\rho_{\mathrm{e}}\left(\frac{\partial}{\partial t}+U_{\mathrm{e}} \frac{\partial}{\partial s}\right)^{2} \eta-\frac{2 B^{2}}{\mu_{0}} \frac{\partial^{2} \eta}{\partial s^{2}}=0$.

A key parameter in our discussion is the arc-length, which is defined as

$$
\frac{\partial s}{\partial \theta}=\sqrt{a(t)^{2} \sin ^{2} \theta+b(t)^{2} \cos ^{2} \theta}=\alpha(\theta, t) .
$$

In order to solve Eq. (20), we would need to express the density as function of $s$ and $t$. However, it turns out that it is much easier to deal with the variable $\theta$ instead. Therefore, we express the derivatives in the governing equation as

$$
\frac{\partial}{\partial s}=\frac{1}{\alpha} \frac{\partial}{\partial \theta}, \quad \frac{\partial^{2}}{\partial s^{2}}=\frac{1}{\alpha^{2}} \frac{\partial^{2}}{\partial \theta^{2}}-\frac{\sin 2 \theta\left(a(t)^{2}-b(t)^{2}\right)}{2 \alpha^{4}} \frac{\partial}{\partial \theta} .
$$

Assuming again a quasi-stationary equilibrium, described in the previous section, and introducing the small parameter, $\epsilon$, the governing equation for transverse kink oscillations can be written as

$$
\begin{aligned}
\frac{\partial^{2} \eta}{\partial t^{2}} & +\frac{2}{\alpha}\left(\frac{\rho_{\mathrm{i}} U_{\mathrm{i}}+\rho_{\mathrm{e}} U_{\mathrm{e}}}{\rho_{\mathrm{i}}+\rho_{\mathrm{e}}}\right) \frac{\partial^{2} \eta}{\partial \theta \partial t}+\frac{1}{\alpha^{2}}\left[\frac{\rho_{\mathrm{i}} U_{\mathrm{i}}^{2}+\rho_{\mathrm{e}} U_{\mathrm{e}}^{2}}{\rho_{\mathrm{i}}+\rho_{\mathrm{e}}}\right. \\
& \left.-\frac{2 \epsilon^{-2} B_{0}^{2}}{\mu_{0}\left(\rho_{\mathrm{i}}+\rho_{\mathrm{e}}\right)}\right] \frac{\partial^{2} \eta}{\partial \theta^{2}}-\left[2 \frac{\rho_{\mathrm{i}} U_{\mathrm{i}}+\rho_{\mathrm{e}} U_{\mathrm{e}}}{\rho_{\mathrm{i}}+\rho_{\mathrm{e}}} \frac{a \dot{a} \sin ^{2} \theta+b \dot{b} \cos ^{2} \theta}{\alpha^{3}}\right. \\
& +\frac{\rho_{\mathrm{i}} U_{\mathrm{i}}^{2}+\rho_{\mathrm{e}} U_{\mathrm{e}}^{2}}{\rho_{\mathrm{i}}+\rho_{\mathrm{e}}} \frac{\sin 2 \theta\left(a(t)^{2}-b(t)^{2}\right)}{2 \alpha^{4}} \\
& \left.-2 \frac{\epsilon^{-2} B_{0}^{2}}{\mu_{0}\left(\rho_{\mathrm{i}}+\rho_{\mathrm{e}}\right)} \frac{\sin 2 \theta\left(a(t)^{2}-b(t)^{2}\right)}{2 \alpha^{4}}\right] \frac{\partial \eta}{\partial \theta}=0
\end{aligned}
$$

which has to be solved subject to the boundary conditions $\eta(\theta=$ $0, \theta=\pi)=0$.

Again, we will solve this equation using the WKB approximation presented earlier and suppose a solution identical 
to Eq. (8). In the first order of approximation we obtain the equation

$$
\frac{\partial^{2} S_{0}}{\partial \theta^{2}}-\frac{\sin 2 \theta\left(a^{2}-b^{2}\right)}{2 \alpha^{2}} \frac{\partial S_{0}}{\partial \theta}+\frac{\alpha^{2} \Omega^{2}}{\tilde{c}_{\mathrm{K}}^{2}} S_{0}=0
$$

together with the usual boundary conditions at the two ends of the loop. In the next order of approximation, we obtain

$$
\begin{aligned}
& \frac{\partial^{2} S_{1}}{\partial \theta^{2}}-\frac{\sin 2 \theta\left(a^{2}-b^{2}\right)}{2 \alpha^{2}} \frac{\partial S_{1}}{\partial \theta}+\frac{\alpha^{2} \Omega^{2}}{\tilde{c}_{\mathrm{K}}^{2}} S_{1}= \\
& \frac{2 \mathrm{i} \alpha \Omega}{\tilde{c}_{\mathrm{K}}^{2}}\left[\frac{\partial S_{0}}{\partial t}+\frac{\alpha S_{0}}{2 \Omega} \frac{\partial \Omega}{\partial t}+\frac{\rho_{\mathrm{i}} U_{\mathrm{i}}+\rho_{\mathrm{e}} U_{\mathrm{e}}}{\rho_{\mathrm{i}}+\rho_{\mathrm{e}}} \frac{\partial S_{0}}{\partial \theta}\right] .
\end{aligned}
$$

This equation has to be solved subject to the boundary condition $S_{1}(\theta=0, \theta=\pi)=0$. Again, the equation for $S_{1}$ will have a solution if the right-hand side of the above equation satisfies the compatibility condition, i.e. the orthogonality to $S_{0}$. Following the same solving procedure as presented earlier, it is easy to show that the condition reduces to

$\omega \alpha \int_{0}^{\pi} \frac{S_{0}^{2}}{c_{\mathrm{K}}^{2}} \mathrm{~d} \theta=0$.

Therefore, the system of Eqs. (24) and (26) will determine completely the dynamics of the expanding coronal loop.

Let us assume that at $t=0$, the loop is semi-circular and its length is $L_{0}$. In order to reproduce the different movement over the two directions, we introduce two different expansion speeds in the horizontal $\left(v_{\mathrm{h}}\right)$ and vertical $\left(v_{\mathrm{v}}\right)$ direction, so that $v_{\mathrm{h}}<v_{\mathrm{v}}$. Again, we suppose that the motion occurs linearly in time and write the dynamics over the two axes as

$x=\left(\frac{L_{0}}{\pi}+v_{\mathrm{h}} t\right) \cos \theta, \quad z=\left(\frac{L_{0}}{\pi}+v_{\mathrm{v}} t\right) \sin \theta$.

Let us introduce a new set of dimensionless quantities

$\tau=\frac{v_{\mathrm{v}} t}{L_{0}}, \quad \tilde{\omega}=\frac{\omega L_{0}}{c_{\mathrm{Kf}}}, \quad \tilde{\alpha}=\frac{\alpha}{L_{0}}, \quad U=\frac{v_{\mathrm{h}}}{v_{\mathrm{v}}}, \quad \tilde{S}_{0}=\frac{S_{0}}{L_{0}}$.

In the new variables, the governing equations become

$$
\begin{aligned}
\frac{\partial^{2} \tilde{S}_{0}}{\partial \theta^{2}} & +\tilde{\alpha}^{2} \tilde{\omega}^{2} \exp \left[-\frac{L_{0}(1+\tau \pi)}{\pi H} \sin \theta\right] \tilde{S}_{0} \\
& +\frac{\sin \theta \cos \theta\left[\tau^{2} \pi^{2}\left(1-U^{2}\right)+2 \tau \pi(1-U)\right]}{1+\tau^{2} \pi^{2}\left(\sin ^{2} \theta+U^{2} \cos ^{2} \theta\right)+2 \tau \pi\left(\sin ^{2} \theta+U \cos ^{2} \theta\right)} \\
& \times \frac{\partial \tilde{S}_{0}}{\partial \theta}=0,
\end{aligned}
$$

and

$\tilde{\omega} \tilde{\alpha} \int_{0}^{\pi}\left(\frac{\tilde{S}_{0}^{2}}{\bar{c}_{\mathrm{K}}^{2}}\right) \mathrm{d} \theta=0$,

where $\bar{c}_{\mathrm{K}}=c_{\mathrm{K}} / v_{\mathrm{v}}$. Figure 4 displays the evolution of the period of oscillations for the fundamental mode and its first harmonic for three different values of $L_{0} / H$, similar values as used in the previous section. Comparing the findings in Figs. 2 and 4, the effect of the expansion into an elliptical shape compared to the constant semi-circular shape is evident. As time progresses, the period of oscillations tends to a higher value for elliptical shape; however, this conclusion is more true for the fundamental mode. The period of the fundamental mode, corresponding to an expansion in a solar corona where scale height is only $23.5 \mathrm{Mm}$

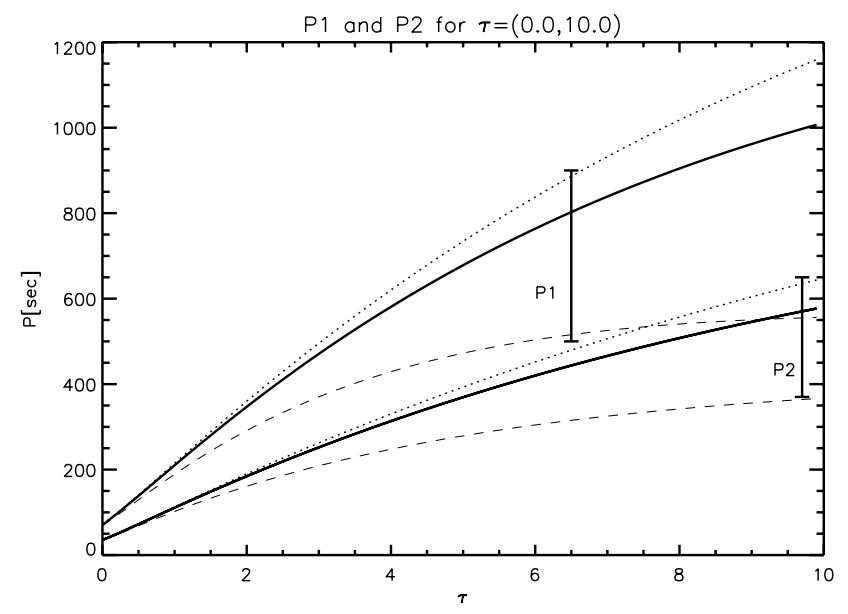

Fig. 4. The same as in Fig. 2, but here we assume that the expansion of the loop occurs such that the loop evolves into a loop with a semi-elliptical shape. The meaning of different line-styles is identical to Fig. 2.

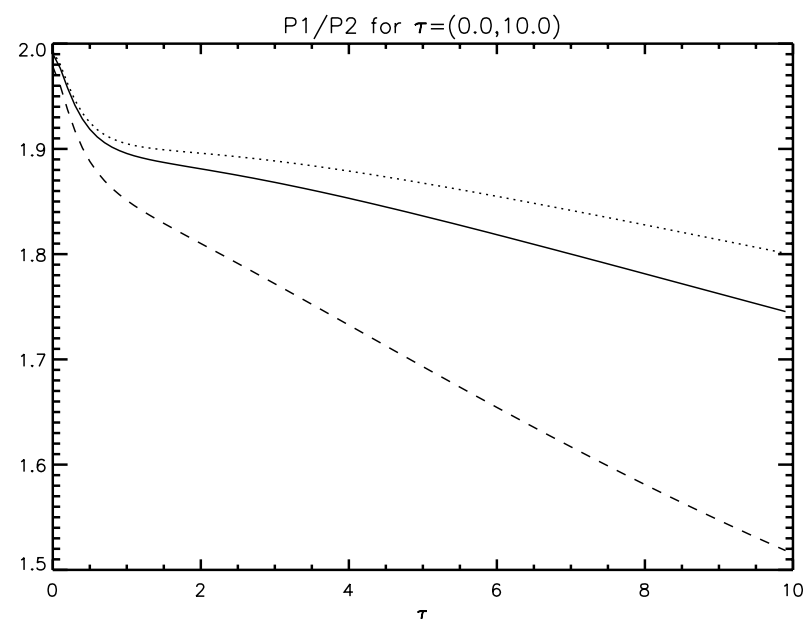

Fig. 5. The same as in Fig. 3, but here we assume that the expansion of the loop occurs such that the loop evolves into a loop with a semi-elliptical shape. The meaning of different line-styles is identical to Fig. 2.

(corresponding to a temperature of $0.5 \mathrm{MK}$ in a loop in hydrostatic equilibrium), saturates rather quickly.

A more significant change is evident when comparing the $P_{1} / P_{2}$ period ratio of the expansion into an elliptical shape (shown in Fig. 5) to the variation of the period ratio for a semicircular shape. For the same time interval, the decrease of the period ratio is much more significant, and, similar to the previous case, the period ratio is more affected for the case of strong stratification, i.e. small $H$.

\section{Conclusions}

The solar corona is a very dynamical environment, where changes in the dynamical state of the plasma and field occur on all sort of time scales. In the present study, we combined for the first time two kinds of dynamical events: the time-evolution of a coronal loop through its expansion into the "empty" corona and the transverse kink oscillations of coronal loops. The emergence and expansion of a coronal loop is a very complex phenomenon, but here we reduced our model to a simplified process, where the expansion is solely described by the change in the length of the loop with an associated temporal equilibrium density variation. 
The governing equation for kink oscillations was solved in the WKB approximation, when the boundary conditions are time-dependent. As expected, due to the change in the length of the loop, the amplitude and periods of oscillations increase with time, while the period ratio of the fundamental mode and its first overtone decreases. This last physical parameter is of paramount importance for the remote determination of density structuring of coronal loops with the help of seismological approaches. In the first instance, we regarded the loop to have an initial semicircular shape that is maintained through the expansion phase. Later, this restriction was lifted based on the natural assumption that the expansion into the vertical direction (i.e. into the direction of density decrease) occurs much more easily than into the horizontal direction. In this limit, the loop evolves into a semiellipse, with the major axis in the vertical direction. Comparing the results of the two approaches, it is clear that the behaviour of the period ratio is sensitive to the geometrical shape of the loop, a more significant drop in the $P_{1} / P_{2}$ ratio being achieved in the second case. Although our numerical results were obtained for three different structuring degrees (measured by the ratio of the initial loop length to the density scale-height), it is also evident that both the temporal change in the loop length and the stratification will have the same effect upon the period ratio, resulting in a mutual amplification of the effect.

Our model predicts that the amplitude of oscillations increases with time, however, due to the particular choice of density, damping processes were neglected. Once the density is allowed to vary also in the radial direction, according to the theory of resonant absorption (e.g. Goossens et al. 1992; Rudeman \& Roberts 2002), loops will damp very quickly with the resonant position displaying a steady motion due to the change of the length of the loop. The amplitude of oscillations can also be damped because the cooling of the plasma (e.g. Morton et al. 2010), an effect that was also neglected here. In an expanding loop, the growth of the amplitude due to emergence and the decay of amplitude owing to resonant damping or cooling will be competing processes. The competition between these two effects will be discussed in a forthcoming paper.

Acknowledgements. I.B. acknowledges the financial support by NFS Hungary (OTKA, K83133). The authors are grateful to Prof M.S. Ruderman and Dr. V. Archontis for their help and comments.

\section{Appendix A: Solutions to the wave equation in the case of the loop at the beginning of its expansion}

An interesting insight into the character of the solution of the governing equation can be obtained analytically in the limiting case of

$\zeta=L_{0}(1+\tau) / \pi H \ll 1$,

i.e. we restrict ourselves to the first part of the emergence into the solar corona. In this case the argument of the exponential function in Eq. (17) can be expanded (keeping only the first two terms) and the equation to be solved reduces to

$\frac{\partial^{2} \tilde{S}_{0}}{\partial \xi^{2}}+\tilde{\omega}^{2} \tilde{S}_{0}=-\sin \frac{\pi \xi}{1+\tau} \zeta \tilde{S}_{0}$

Since we are looking for periodic solutions and expect that both the amplitude and frequency will depend on time, we will employ the Poincaré-Lindstedt method (Meirovitch 1970) to find corrections to the eigenfunctions and eigenfrequencies (it can be easily shown that this method is similar to the re-normalization technique used by Ballai et al. 2007, in the case of dispersive shocks). We are looking for solutions in the form of series and write

$\tilde{S}_{0}=\sum_{k=1}^{\infty} \zeta^{k} \tilde{S}_{0}^{(k)}, \quad \tilde{\omega}=\sum_{k=1}^{\infty} \zeta^{k} \tilde{\omega}^{(k)}$

where the functions $S_{\mathrm{i}}$ are periodic functions. We first concentrate on the fundamental mode. After inserting the expansions (A.2) into the governing Eq. (A.1), we collect terms proportional to subsequent powers of $\zeta$. In the first order of approximation, collecting terms $\sim O\left(\zeta^{0}\right)$, results in

$\frac{\partial^{2} \tilde{S}_{0}^{(0)}}{\partial \xi^{2}}+\tilde{\omega}^{(0) 2} \tilde{S}_{0}^{(0)}=0$.

Solving this equation, subject to the aforementioned boundary conditions, yields in the case of the fundamental mode

$\tilde{S}_{0}^{(0)} \sim \sin \frac{\pi \xi}{1+\tau}, \quad \tilde{\omega}^{(0)}=\frac{\pi}{1+\tau}$.

In the next order of approximation (i.e. terms $\sim O(\zeta)$ ) we obtain

$\frac{\partial^{2} \tilde{S}_{0}^{(1)}}{\partial \xi^{2}}+\tilde{\omega}^{(0) 2} \tilde{S}_{0}^{(1)}=-\frac{\pi^{2}}{(1+\tau)^{2}} \sin ^{2} \frac{\pi \xi}{1+\tau}-\frac{2 \pi}{1+\tau} \tilde{\omega}^{(1)} \sin \frac{\pi \xi}{1+\tau}$,

which has to be solved subject to the boundary conditions

$\tilde{S}_{0}^{(1)}(\xi=0, \xi=1+\eta)=0$.

The last term in Eq. (A.5) will cause secular growth of the solution, rendering the solution $S_{1}$ nonperiodic. To suppress this possibility, we choose $\tilde{\omega}_{1}=0$. As a result, the solution of Eq. (A.5) together with the boundary conditions is simply written as

$\tilde{S}_{0}^{(1)} \sim \sin \frac{\pi \xi}{1+\tau}+\frac{3}{2} \cos \frac{\pi \xi}{1+\tau}-\frac{1}{6}\left(3+\cos \frac{2 \pi \xi}{1+\tau}\right)$.

In the next order of approximation, we collect terms $O\left(\zeta^{2}\right)$ and obtain

$\frac{\partial \tilde{S}_{0}^{(2)}}{\partial \xi}+\tilde{\omega}^{(0) 2} \tilde{S}_{0}^{(2)}=-\tilde{\omega}^{(0) 2} \tilde{S}_{0}^{(1)} \sin \left(\frac{\pi \xi}{1+\tau}\right)-2 \tilde{\omega}^{(0)} \tilde{\omega}^{(1)} \tilde{S}_{0}^{(0)}$.

Using the expression of $\tilde{\omega}^{(0)}, \tilde{S}_{0}^{(0)}$ and $\tilde{S}_{0}^{(1)}$ determined earlier, the RHS of the above equation can be written as

$$
\begin{aligned}
R H S= & {\left[\frac{\pi^{2}}{3(1+\tau)^{2}}-\frac{2 \pi \tilde{\omega}^{(2)}}{1+\tau}\right] \sin \frac{\pi \xi}{1+\tau}+A \sin ^{2} \frac{\pi \xi}{1+\tau} } \\
& +B \sin \frac{2 \pi \xi}{1+\tau}+C \sin \frac{3 \pi \xi}{1+\tau}
\end{aligned}
$$

where the coefficients of higher harmonics $(A, B$, and $C)$ are not needed for our discussion. In order to prevent nonperiodic behaviour, we need to impose the condition that the coefficient of the first term is zero, leading to

$\tilde{\omega}^{(2)}=\frac{\pi}{6(1+\tau)} \cdot$

As a result, the eigenfunction and eigenfrequency of fundamental mode oscillations can be written as

$\tilde{S}_{0}=\sin \frac{\pi \xi}{1+\tau}+\zeta\left[\frac{3}{2} \cos \frac{\pi \xi}{1+\tau}-\frac{1}{6}\left(3+\cos \frac{2 \pi \xi}{1+\tau}\right)\right]$,

$\tilde{\omega}=\frac{\pi}{1+\tau}+\zeta^{2} \frac{\pi}{6(1+\tau)}$, 
meaning that the change in the frequency due to expansion is a second-order effect. It is easy to show that the period of the fundamental mode in this approximation can be written as

$P_{1}=\frac{12(1+\tau)}{6+\zeta^{2}}$

proving the increase of the period $P_{1}$ with $\tau$ seen in Fig. 2 .

Repeating the same method for the first harmonic, where the eigenfunction and eigenfrequency in the zeroth-order approximation are

$\tilde{S}_{0}=\sin \frac{2 \pi \xi}{1+\tau}, \quad \tilde{\omega}^{(0)}=\frac{2 \pi}{1+\tau}$,

we obtain that

$\tilde{\omega}=\frac{2 \pi}{1+\tau}+\zeta^{2} \frac{2 \pi}{15(1+\tau)}$,

meaning that the period of the first harmonic in this approximation behaves like

$P_{2}=\frac{15(1+\tau)}{15+\zeta^{2}}$

showing an increasing tendency with respect to $\tau$. Now, using Eqs. (A.12) and (A.14), we can calculate the period ratio of the fundamental mode and its first harmonic as

$\frac{P_{1}}{P_{2}} \approx 2\left(1-\frac{\zeta^{2}}{60}\right)=2\left[1-\frac{L_{0}^{2}(1+\tau)^{2}}{60 \pi^{2} H^{2}}\right]$,

so that the change in the period ratio is very small but decreases with $\zeta$.

\section{References}

Andries, J., Arregui, I., \& Goossens, M. 2005, ApJ, 624, L57

Andries, J., van Doorsselaere, T., Roberts, B., et al. 2009, Space Sci. Rev., 149, 3 Archontis, V. 2008, J. Geophys. Res., 113, A03S04

Archontis, V., Moreno-Insertis, F., Galsgaard, K., et al. 2004, A\&A, 426, 1047 Arregui, I., Oliver, R., \& Ballester, J. L. 2012, Liv. Rev. Sol. Phys., 9, 2
Aschwanden, M. J. 2004, Physics of the Solar Corona (Berlin: Springer-Verlag) Ballai, I. 2007, Sol. Phys., 246, 177

Ballai, I., Erdélyi, R., \& Pintér, B. 2005, ApJ, 633, L145

Ballai, I., Forgács-Dajka, E., \& Marcu, A. 2007a, Astron. Nachr., 328, 734

Ballai, I., Jess, D., \& Douglas, M. 2007b, A\&A, 534, A13

Ballai, I., Douglas, M., \& Marcu, A. 2008, A\&A, 488, 1125

Banerjee, D., Erdélyi, R., Oliver, R., \& O’Shea, E. 2007, Sol. Phys., 246, 3

Bender, M., \& Ország, S. A. 1987, Advanced Mathematical Methods for Scientists and Engineers (Singapore: McGraw-Hill)

Chou, D.-Y., \& Zirin, H. 1988, ApJ, 333, 420

Diaz, A. J., Donelly, G. R., \& Roberts, B. 2007, A\&A, 476, 359

Dymova, M. V., \& Ruderman, M. S. 2006, A\&A, 457, 1069

Eto, S., Isobe, H., Narukage, N., et al. 2002, PASJ, 54, 481

Goossens, M., Hollweg, J. V., \& Sakurai, T. 1992, Sol. Phys., 138, 233

Harra, L. K., Magara, T., Hara, H., et al. 2010, Sol. Phys., 263, 105

Harra, L. K., Archontis, V., Pedran, E., et al. 2012, Sol. Phys., 278, 47

Isobe, H., \& Tripathi, D. 2007, A\&A, 449, L17

Jing, J., Lee, J., Spirock, T. J., et al. 2003, ApJ, 584, L103

McEwan, M. P., Donnelly, G. R., Díaz, A. J., \& Roberts, B. 2006, A\&A, 460, 893

McEwan, M. P., Díaz, A. J., \& Roberts, B. 2008, A\&A, 481, 819

Meirovitch, L. 1970, Methods of Analytical Dynamics (Michigan, USA: McGraw-Hill)

Morton, R. J., \& Erdélyi, R. 2009, A\&A, 502, 315

Morton, R. J., Hood, A. W., \& Erdélyi, R. 2010, A\&A, 512, A23

Morton, R. J., Ruderman, M. S., \& Erdélyi, R. 2011, A\&A, 534, A27

Nakariakov, V. M., Ofman, L., Deluca, E. E., Roberts, B., \& Davila, J. M. 1999, Science, 285, 862

Okamoto, T. J., Nakai, H., \& Keiyama, A. 2004, ApJ, 608, 1124

Orza, B., Ballai, I., Jain, R., \& Murawski, K. 2012, A\&A, 537, A41

Parker, E. N. 1955, ApJ, 122, 293

Parker, E. N. 1988, ApJ, 326, 407

Pintér, B., Jain, R., Tripathi, D., \& Isobe, H. 2008, ApJ, 680, 1560

Ramsey, H. E., \& Smith, S. F. 1966, AJ, 71, 197

Roberts, B., Edwin, P. M., \& Benz, A. O. 1984, ApJ, 279, 857

Ruderman, M. S. 2010, Sol. Phys., 267, 377

Ruderman, M. S. 2011a, Sol. Phys., 271, 41

Ruderman, M. S. 2011b, A\&A, 534, A78

Ruderman, M. S., \& Erdélyi, R. 2009, Space Sci. Rev., 149, 199

Ruderman, M. S., \& Roberts, B. 2002, ApJ, 577, 475

Ruderman, M. S., Verth, G., \& Erdélyi, R. 2008, ApJ, 686, 694

Schmidt, J. M., \& Ofman, L. 2011, ApJ, 739, 75

Tomczyk, S., McIntosh, S. W., Keil, S. L., et al. 2007, Science, 317, 1192

Verth, G., \& Erdélyi, R. 2008, A\&A, 486, 1015

Verth, G., Van Doorsselaere, T., Erdélyi, R., \& Goossens, M. 2007, A\&A, 475, 341

Zwaan, C. 1987, ARA\&A, 25, 83

Wang, T., Ofman, L., Davila, J., et al. 2012, ApJ, 751, 27 